\title{
Signal Uncertainty in Spectrum Sensing for Cognitive Radio
}

\author{
Miguel López-Benítez, Member, IEEE, Fernando Casadevall, Member, IEEE
}

\begin{abstract}
The inability to perfectly know the system noise properties to infinite precision, referred to as noise uncertainty, results in noise power calibration errors that have been proven to impose fundamental limitations on the detection performance of any spectrum sensing (signal detection) method in cognitive radio networks. In this work we argue that the inability of cognitive radio users to perfectly know beforehand the primary signals that might be present in the sensed band and their properties, referred to as signal uncertainty in this work, also results in an additional detection performance degradation. The noise uncertainty consequences have widely been studied, verified experimentally and distilled into tractable mathematical models. However, the potential effects of the particular primary signal properties on the resulting detection probability of generic spectrum sensing algorithms, such as energy detection, have not been taken into account in the analysis and performance evaluation of spectrum sensing in cognitive radio networks. In this context, this work develops a mathematical model for signal uncertainty and, based on such model, analyzes the impact of signal uncertainty on the resulting detection performance of spectrum sensing, with and without noise uncertainty, and compares the practical consequences of both degrading effects.
\end{abstract}

Index Terms-Cognitive radio, spectrum sensing, energy detection, noise uncertainty, signal uncertainty.

\section{INTRODUCTION}

W HILE still early in its deployment, Dynamic Spectrum Access/Cognitive Radio (DSA/CR) [1]-[3] is a highly promising communications paradigm that can effectively address the spectrum insufficiency problem, which arises as a result of the existing conflicts between spectrum demand growth and spectrum underutilization. DSA/CR aims at improving spectrum usage efficiency by allowing some unlicensed (secondary) users to access in an opportunistic and non-interfering manner some licensed bands temporarily unoccupied by the licensed (primary) users.

One of the most important challenges for a DSA/CR network is not to cause harmful interference to primary users. To guarantee interference-free spectrum access, secondary users should reliably identify spectrum opportunities, which

Paper approved by M. Buehrer, the Editor for Cognitive Radio and UWB of the IEEE Communications Society. Manuscript received April 16, 2012; revised October 11, 2012.

M. López-Benítez was with the Department of Signal Theory and Communications, Universitat Politècnica de Catalunya, Barcelona, Spain. He currently is with the Centre for Communication Systems Research (CCSR), University of Surrey, Guildford, United Kingdom (email: m.lopez@surrey.ac.uk).

F. Casadevall is with the Department of Signal Theory and Communications, Universitat Politècnica de Catalunya, Barcelona, Spain (email: ferranc@tsc.upc.edu).

This work was supported by the Spanish Research Council under ARCO grant (Ref. TEC2010-15198) and FPU grant (Ref. AP2006-848).

Digital Object Identifier 10.1109/TCOMM.2013.XX.XXXXXX basically means being able to determine whether a primary signal is present within a certain frequency range [4]. A number of different signal detection methods, referred to as spectrum sensing algorithms in the context of DSA/CR, have been proposed in the literature to identify the presence of primary signal transmissions [5]-[7]. The existing solutions provide different trade-offs between required sensing time, complexity and detection capabilities, but their practical applicability depends on how much information is available about the primary user signal. In the most generic case, a DSA/CR user is not expected to be provided with any prior information about the primary signals that may be present within a certain frequency band. When the secondary receiver cannot gather sufficient information, the Energy Detection (ED) principle [8] can be used due to its ability to work irrespective of the signal format to detect. Despite its practical performance limitations, ED has gained popularity as a spectrum sensing technique for DSA/CR due to its general applicability and simplicity as well as its low computational and implementation costs. ED has been a preferred approach for many past spectrum sensing studies and also constitutes the spectrum sensing method considered in this work.

A recent study [9] based on empirical measurements of real signals demonstrated that the detection performance of ED may strongly vary with the primary radio technology being detected. Certain technology-dependent inherent properties may result in different detection performances for various primary signals under the same conditions. In other words, the detection probability for a fixed set of operating parameters might be enough to reliably detect some primary signals but might not for some others, thus making some radio technologies more susceptible to interferences under the same operating conditions. The inability of a DSA/CR user to perfectly know beforehand the primary signals that might be present in the sensed band and their properties, referred to as signal uncertainty in this work, results in a detection performance degradation since the non-interference requirement for the secondary network implies a worst-case design where secondary users must guarantee a minimum detection performance regardless of the sensed primary signal.

While the consequences of noise uncertainty (i.e., the inability to perfectly calibrate the system noise) have widely been studied, experimentally verified and mathematically modeled [10], [11], the impact of signal uncertainty on the detection performance of spectrum sensing, which may have important practical consequences as experimentally demonstrated in [9], has not been studied and analyzed yet. The existing classical theoretical results for ED describe the resulting probability of 
detection when the received primary signal is impaired by Additive White Gaussian Noise (AWGN) or wireless propagation environments such as Nakagami or Rayleigh fading channels [12]. Nevertheless, the potential effect of the particular primary signal properties on the resulting detection probability of generic spectrum sensing algorithms, such as ED, has not been taken into account in the analysis and performance evaluation of spectrum sensing in DSA/CR networks. In this context, this work covers such deficiencies and fills the existing gaps by developing a model for signal uncertainty and analyzing its impact on the resulting detection performance of ED. First, Section II reviews the operating principle and theoretical fundamentals of ED under AWGN channels as well as the consequences of noise uncertainty. Starting with these classical theoretical results, and after highlighting its necessity in Section III, a model for signal uncertainty is developed in Section IV based on theoretical reasonings complemented with empirical approximations. Concretely, a generic mathematical expression for the received Signal-to-Noise Ratio (SNR) distribution under variable primary transmission power patterns is developed, which is then employed to derive the average detection probability of ED under signal uncertainty. Based on such model, Section V discusses and exemplifies with numerical results the impact of signal uncertainty on the detection performance of ED, and compares the degrading effect of signal uncertainty with that of noise uncertainty. Finally, Section VI summarizes the main conclusions derived from this work.

\section{THEORETICAL PERFORMANCE OF ENERGY DETECTION}

\section{A. Spectrum sensing}

The spectrum sensing problem can be formulated as a binary hypothesis testing problem with the following two hypotheses:

$$
\begin{array}{ll}
\mathcal{H}_{0}: y[n]=w[n] & n=1,2, \ldots, N \\
\mathcal{H}_{1}: y[n]=x[n]+w[n] & n=1,2, \ldots, N
\end{array}
$$

where $\mathcal{H}_{0}$ is a null hypothesis stating that the received signal samples $y[n]$ correspond to noise samples $w[n]$ and therefore there is no primary signal in the sensed spectrum band, and hypothesis $\mathcal{H}_{1}$ indicates that some licensed user signal $x[n]$ is present. $N$ denotes the number of samples collected during the signal observation interval (i.e., the sensing period), emphasizing that the decision is made based on a limited number of signal samples. The ideal spectrum sensor would select hypothesis $\mathcal{H}_{1}$ whenever a primary signal is present and hypothesis $\mathcal{H}_{0}$ otherwise. Unfortunately, spectrum sensing algorithms may fall into mistakes in practice, which can be classified into miss detections and false alarms. A miss detection occurs when a primary signal is present in the sensed band and the spectrum sensing algorithm selects hypothesis $\mathcal{H}_{0}$, which may result in harmful interference to primary users. On the other hand, a false alarm occurs when the sensed spectrum band is idle and the spectrum sensing algorithm selects hypothesis $\mathcal{H}_{1}$, which results in missed transmission opportunities and therefore in a lower spectrum utilization. Based on these definitions, the performance of any spectrum sensing algorithm can be summarized by means of two probabilities: the probability of miss detection $P_{m d}=P\left(\mathcal{H}_{0} / \mathcal{H}_{1}\right)$, or its complementary probability of detection $P_{d}=P\left(\mathcal{H}_{1} / \mathcal{H}_{1}\right)=1-P_{m d}$, and the probability of false alarm $P_{f a}=P\left(\mathcal{H}_{1} / \mathcal{H}_{0}\right)$. Large $P_{d}$ and low $P_{f a}$ values would be desirable. Nevertheless, there exists a trade-off between $P_{d}$ and $P_{f a}$, meaning that improving one of the performance metrics implies degrading the other one.

\section{B. Energy detection}

The ED principle, also referred to as radiometric detection, measures the energy received on a primary band during an observation interval and declares the band as busy if the measured energy is greater than a properly set predefined threshold, or idle otherwise [8]:

$$
\mathbb{T}(\mathbf{y})=\sum_{n=1}^{N}|y[n]|^{2} \underset{\mathcal{H}_{0}}{\stackrel{\mathcal{H}_{1}}{\gtrless}} \lambda
$$

where $\mathbb{T}(\mathbf{y})$ is the ED test statistic computed over the signal vector $\mathbf{y}=(y[1], y[2], \ldots, y[N])$, and $\lambda$ is a fixed decision threshold to distinguish between the two hypotheses in (1).

The decision threshold $\lambda$ could be chosen for an optimum trade-off between $P_{d}$ and $P_{f a}$. However, this would require knowledge of the noise and detected signal powers. While the noise power can be estimated with relatively simple methods [13], the estimation of the signal power, which depends on many varying factors such as transmission and propagation characteristics, involves the use of complex and computationally costly methods. In practice, the threshold is normally chosen to satisfy a certain $P_{f a}$ [14], which only requires the noise power to be known. For a Constant False-Alarm Rate (CFAR) threshold-setting approach [14], the detection probability of ED under AWGN channels as a function of the SNR, denoted as $\gamma$, is given by [15]:

$$
\begin{aligned}
P_{d}(\gamma) & =\mathcal{Q}\left(\frac{\mathcal{Q}^{-1}\left(P_{f a}\right) \sqrt{2 N}-N \gamma}{\sqrt{2 N}(1+\gamma)}\right) \\
& \approx \mathcal{Q}\left(\mathcal{Q}^{-1}\left(P_{f a}\right)-\sqrt{\frac{N}{2} \gamma}\right)
\end{aligned}
$$

where $\mathcal{Q}(\cdot)$ is the standard Gaussian tail probability $Q$ function [16, (26.2.3)]. The approximation of (3b) assumes the case of low SNR regime in DSA/CR $(\gamma \ll 1)$.

\section{Noise uncertainty}

Calibration errors as well as changes in thermal noise caused by temperature variations limit the accuracy to which noise power can be estimated, leading to some noise uncertainty. In practice, the noise power is therefore uncertain within a certain interval [17]. Let's assume the noise uncertainty model $\hat{\sigma}_{w}^{2} \in\left[\sigma_{w}^{2}, \alpha \sigma_{w}^{2}\right][18]$, where $\hat{\sigma}_{w}^{2}$ represents the estimated noise power, $\sigma_{w}^{2}$ is the nominal noise power and $\alpha>1$ is the noise uncertainty. Based on the estimated noise power $\hat{\sigma}_{w}^{2}$, the worstcase $\left(\hat{\sigma}_{w}^{2}=\alpha \sigma_{w}^{2}\right)$ detection probability given by (3) becomes:

$$
\begin{aligned}
P_{d}(\gamma) & =\mathcal{Q}\left(\frac{\alpha \mathcal{Q}^{-1}\left(P_{f a}\right) \sqrt{2 N}-N(\gamma+1-\alpha)}{\sqrt{2 N}(1+\gamma)}\right) \\
& \approx \mathcal{Q}\left(\alpha \mathcal{Q}^{-1}\left(P_{f a}\right)-\sqrt{\frac{N}{2}}(\gamma+1-\alpha)\right)
\end{aligned}
$$




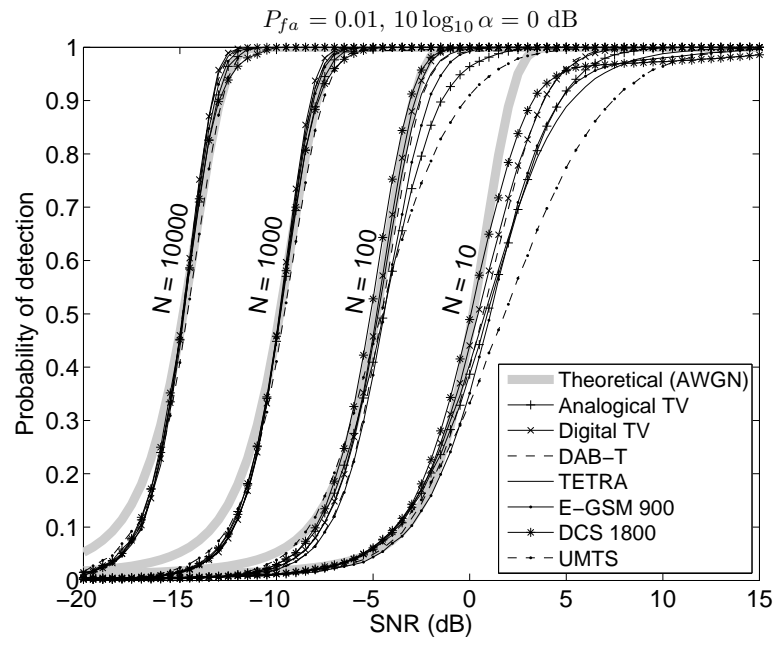

Fig. 1. Theoretical and experimental performance of energy detection without noise uncertainty.

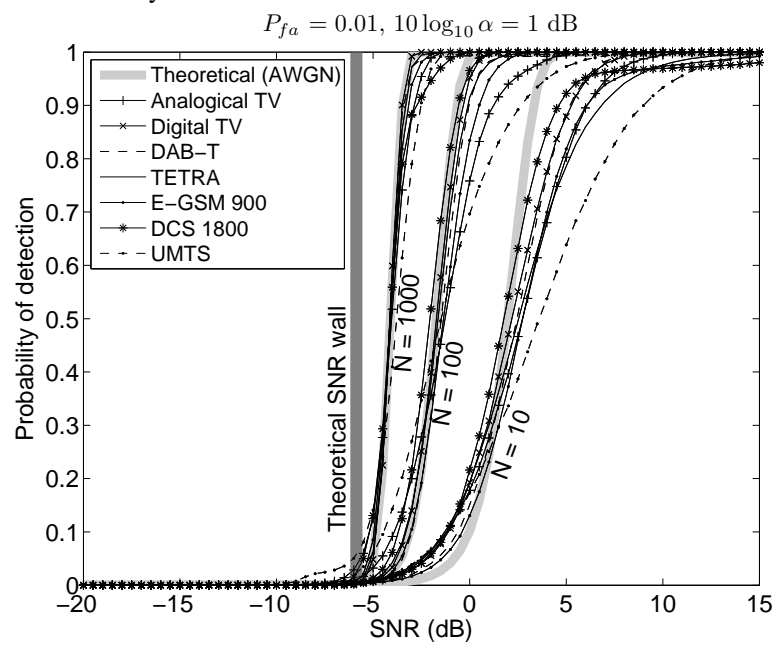

Fig. 2. Theoretical and experimental performance of energy detection with 1 -dB noise uncertainty.

Equations (3) and (4) are graphically shown as light thick lines in Figures 1 and 2, respectively. As appreciated, noise uncertainty imposes fundamental limitations on detection performance. Although classical detection theory states that degradation in the detection performance due to reduced SNR can be countered by increasing the sensing time [19], [20] (see Figure 1), in practice there exists a limit referred to as $S N R$ wall (see Figure 2), which is given by $\gamma_{\text {wall }}=\alpha-1$ [18], below which the primary signal cannot reliably be detected no matter how long the sensing period is [10], [11]. The existence of SNR walls has been verified experimentally [9], [21], [22], demonstrating that a small noise power estimation error may cause significant performance loss in ED.

\section{EMPIRICAL PERFORMANCE OF ENERGY DETECTION}

Figures 1 and 2 show the experimental performance of the ED method when applied to real-world primary signals of various radio technologies, including analogical and digital TV, DAB-T, TETRA, E-GSM 900, DCS 1800 and UMTS. A detailed and in-depth description of the measurement platform as well as the measurement and evaluation methodologies employed to obtain these results can be found in [9].

Two important aspects in Figures 1 and 2 are noteworthy. First, the experimental results indicate that the ED performance may notably vary with the radio technology being detected. This behavior is not predicted by the classical theoretical results associated to ED, which were presented in Section II. As a matter of fact, for a given set of operating parameters (target $P_{f a}$, sample length $N$, SNR $\gamma$ and noise uncertainty $\alpha$ ) the expressions in (3) and (4) suggest that the resulting performance in terms of $P_{d}$ is unique. However, Figures 1 and 2 clearly demonstrate that the experimental $P_{d}$ may strongly depend on the primary signal being sensed. Second, the performance differences among various radio technologies are not constant, but depend on the sensing period $N$. Summarizing the analysis and discussion of [9], this behavior can be explained as follows. If $N$ is sufficiently low, the ED test statistic may follow the instantaneous variations of the received signal energy. Under the same average SNR conditions (i.e., signals with the same average energy, assuming constant average noise energy), this means that a higher signal energy variability (variance) implies a higher probability that the instantaneous energy level (and the ED test statistic) falls below the decision threshold. In such a case, the channel would be declared as idle even if it should be declared as busy, thus resulting in a degraded detection performance. Since various radio technologies may exhibit different signal energy variation patterns and variances, this explains the different detection performances observed in Figures 1 and 2. As $N$ increases, the test statistics are computed over longer observation periods, thus averaging the peculiarities of any instantaneous energy variation pattern and reducing its variance. In such a case, although the variability of the received energy remains the same, the variability of the test statistic decreases and so does the probability of misdetecting the primary signal. For sufficiently long observation periods, the test statistic ceases to follow the instantaneous signal energy variations and its value closely resembles the true signal energy. When this occurs for all the considered signals, the obtained performance curves converge. This explains the convergent trend observed in Figures 1 and 2 as $N$ increases.

The discussion above indicates that the variability (variance) of the primary transmission power pattern may notably affect the ED performance ${ }^{1}$. The potential effect of this particular primary signal property on the resulting detection performance has not been studied and cannot be predicted with the existing analytical results. A novel model is developed in Section IV.

\footnotetext{
${ }^{1}$ The variability of the received power might be ascribed to the propagation environment where the measurements were performed and the fading properties of the channel. However, it is worth noting that the measurement conditions were carefully selected to minimize the impact of fading and the empirical data were carefully analyzed and selected to ensure that the transmission power pattern was the dominant aspect in the variability of the received power (see [9] for details on the measurements and methodological aspects). In fact, the resulting detection performance for signals with perfectly constant transmission power patterns (e.g., digital TV) agrees with the theoretical prediction for an AWGN channel (i.e., a channel with no fading where the SNR is constant). Therefore, the variation of the received power due to the channel fading was not significant in the field measurements.
} 


\section{Signal UnCERTAinty MOdEL}

\section{A. Problem formulation}

In practice, the SNR is not constant at the secondary receiver since it is the result of the combined effects of the primary transmission power pattern and the propagation environment. As a result, the probability of detection instantaneously varies with the receiving SNR. In such a case, (4) gives the probability of detection $P_{d}$ conditioned on the instantaneous value of $\gamma$. Under varying SNR, a more useful performance parameter is the average probability of detection $\bar{P}_{d}$ experienced for an average SNR $\gamma_{0}$, which may be derived by averaging (4) over the SNR statistics:

$$
\bar{P}_{d}\left(\gamma_{0}\right)=\mathbb{E}\left[P_{d}(\gamma)\right]=\int_{\gamma} P_{d}(\gamma) f_{\gamma}(\gamma) d \gamma
$$

where $P_{d}(\gamma)$ is given by (4) and $f_{\gamma}(\gamma)$ is the Probability Density Function (PDF) of the received SNR. Closed-form expressions for the integral in (5) under Nakagami and Rayleigh fading environments have been derived in the literature [12] in order to reflect the impact of radio propagation channels on the resulting detection performance of ED. In contrast, the focus of this work is on the impact of the primary transmission power pattern. Therefore, it will be assumed that $f_{\gamma}(\gamma)$ is mostly the result of the primary transmission power pattern, which was shown in Section III and demonstrated in [9] to strongly impact the ED performance. The particular shape of $f_{\gamma}(\gamma)$, and hence the detection performance $\bar{P}_{d}\left(\gamma_{0}\right)$, will depend on the primary transmission power pattern and its variability (variance). The main objective of this section is to solve (5) to establish a direct relation between the primary power variability and the resulting detection performance of ED. To this end, a model for $f_{\gamma}(\gamma)$ is required, which is developed in Section IV-B.

\section{B. Approximation for the SNR statistics}

From a realistic point of view, it is reasonable to assume that $f_{\gamma}(\gamma)$ cannot be perfectly known in practice. Determining an exact expression for the distribution of received SNR values, $f_{\gamma}(\gamma)$, would require a perfect knowledge of the transmission power statistics, which are in general unknown and cannot easily be determined in reality since they may depend on many factors such as the particular radio technology being detected and its signaling format as well as the instantaneous conditions of the primary network (e.g., a cellular mobile communication system with load-based power control). Even if the transmission power statistics of any primary radio technology could be assumed to be known, the obtained expression for $f_{\gamma}(\gamma)$ would be valid for a single radio technology, thus requiring a separate analysis for every possible primary radio technology to be detected. Such analysis would result impractical.

As an alternative, and given that deriving an individual analytical expression of $f_{\gamma}(\gamma)$ for every possible case is infeasible, this section is aimed at developing a general, technology-independent approximated expression that can be determined without a perfect knowledge of the primary transmission power statistics and that can be used to describe the distribution of the received SNR values over a wide range of cases with a reasonable level of accuracy. This approach would be valid as long as the resulting expression in (5) is able to describe, with a sufficient level of accuracy, the experimental probability of detection and the performance differences observed in Figures 1 and 2 for real-world signals of different radio technologies.

In order to find a realistic model for $f_{\gamma}(\gamma)$, the empirical SNR PDFs of various real-world signals were computed based on the measurements performed in [9] for different radio technologies (analogical/digital TV, DAB-T, TETRA, E-GSM 900, DCS 1800 and UMTS). For each individual signal of each measured radio technology, the obtained empirical PDF was compared to a wide range of PDF models (Figure 3 shows some examples for selected signals). It was found that most of the empirical PDFs could acceptably be approximated by either Rayleigh distributions $f_{\gamma}^{R}(\gamma)=\left(\gamma / s^{2}\right) \exp \left(-\gamma^{2} / 2 s^{2}\right)$, $\gamma \geq 0$ (Figure 3(a) shows an example) or gamma distributions $f_{\gamma}^{G}(\gamma)=\left(\gamma^{k-1} / \theta^{k} \Gamma(k)\right) \exp (-\gamma / \theta), \gamma \geq 0$, with $k=2$ (Figure 3(b) shows an example). For these distributions, the parameters $s$ and $\theta$ can be computed as $s=\sqrt{2 / \pi} \gamma_{0}=$ $\sqrt{2 \sigma_{\gamma}^{2} /(4-\pi)}$ and $\theta=\gamma_{0} / 2=\sqrt{\sigma_{\gamma}^{2} / 2}$ [23], where $\gamma_{0}$ and $\sigma_{\gamma}^{2}$ represent the average SNR and the SNR variance, respectively. The values of $\gamma_{0}$ and $\sigma_{\gamma}^{2}$ for the captured signals were respectively estimated as the sample mean and sample variance of the instantaneously received SNR values after normalizing the signal sequences as detailed in [9]. Based on such estimations, the corresponding PDF models were derived based on either $\gamma_{0}$ (denoted as mean-based) or $\sigma_{\gamma}^{2}$ (denoted as variance-based). As it can be observed in Figures 3(a) and 3(b), the Rayleigh and gamma distributions provide reasonably good fits for empirical SNR PDFs observed in reality and could be considered as models for the SNR distribution $f_{\gamma}(\gamma)$.

For the Rayleigh and gamma distributions, the SNR mean and variance are related as $\sigma_{\gamma}^{2}=(4 / \pi-1) \gamma_{0}^{2} \approx 0.27 \gamma_{0}^{2}$ for the former and $\sigma_{\gamma}^{2}=0.5 \gamma_{0}^{2}$ for the latter [23]. This indicates the existence of a relation of the form $\sigma_{\gamma}^{2}=\beta \gamma_{0}^{2}$ in practice, where $\beta=\sigma_{\gamma}^{2} / \gamma_{0}^{2}>0$ can be regarded as a normalized variance and thus as a metric representing the variability of the primary transmission power pattern. As mentioned before, the aim is to establish a direct relation between the primary power variability, which can be quantified by means of $\beta$, and the resulting detection performance $\bar{P}_{d}\left(\gamma_{0}\right)$. In the case of the Rayleigh and gamma distributions, the value of $\beta$ is fixed ( $\beta \approx 0.27$ for the former, $\beta=0.5$ for the latter). This means that the resulting expression for $\bar{P}_{d}\left(\gamma_{0}\right)$ in such cases would not allow to analyze the ED performance for any arbitrary primary power variability $\beta$, but only for those cases where the received SNR can adequately be modeled with a Rayleigh or gamma distribution. This observation motivates the search of an alternative model for $f_{\gamma}(\gamma)$ where $\beta$ can be configured in order to analyze the impact of arbitrary primary power variabilities (variances) on the detection performance $\bar{P}_{d}\left(\gamma_{0}\right)$. A simple and analytically tractable PDF model verifying this property is the Gaussian distribution, where the variance is independent of the mean. It is important to note, however, that the Gaussian PDF is defined for any real value while $\gamma \geq 0$. This issue can be resolved by truncating the Gaussian PDF to 


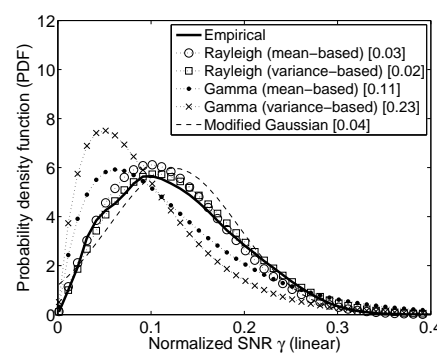

(a)

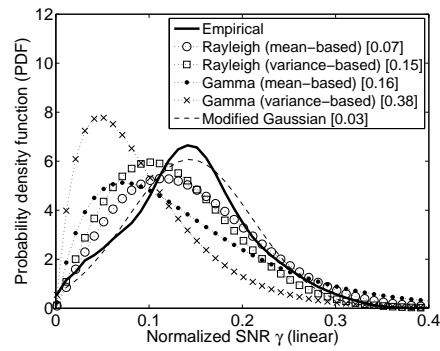

(c)

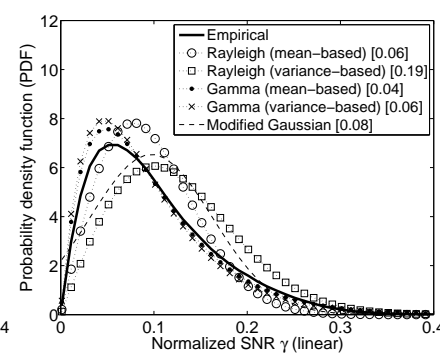

(b)

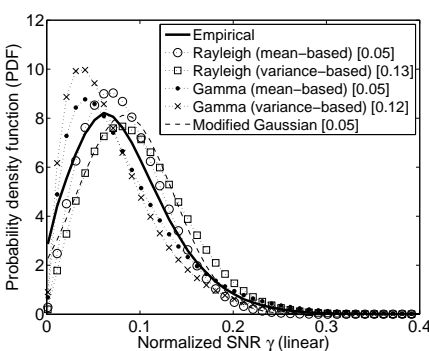

(d)
Fig. 3. Examples of empirical and approximated PDFs of the received SNR for selected signals from field measurements (numbers between square brackets represent the Kolmogorov-Smirnov distance to the empirical distribution).

positive values and introducing a normalization factor $\kappa$ such that $\int_{0}^{\infty} f_{\gamma}(\gamma) d \gamma=1$. This results in the following SNR PDF model [24, Appendix A]:

$$
f_{\gamma}^{M G}(\gamma) \approx \frac{\kappa}{\sqrt{2 \pi} \sigma_{\gamma}} e^{-\frac{1}{2}\left(\frac{\gamma-\gamma_{0}}{\sigma_{\gamma}}\right)^{2}}, \quad \gamma \geq 0
$$

where the normalization factor is given by $\kappa=2 /[1+$ $\left.\operatorname{erf}\left(\gamma_{0} / \sqrt{2} \sigma_{\gamma}\right)\right]$, with $\operatorname{erf}(\cdot)$ being the error function [16, (7.1.1)]. While $\sigma_{\gamma}^{2}$ and $\gamma_{0}$ seem to be mathematically independent parameters in (6), the analysis of empirical data reveals that the relation $\sigma_{\gamma}^{2}=\beta \gamma_{0}^{2}$ (with $\beta>0$ ) also holds here. Note that $\sigma_{\gamma}^{2}$ and $\gamma_{0}$ cannot be independent in practice as a result of the bound $\gamma \geq 0$ (e.g., a low mean value $\gamma_{0}$ cannot be associated with an arbitrarily large variance $\sigma_{\gamma}^{2}$ ). The main advantage of this model with respect to the Rayleigh and gamma models is that the resulting expression for $\bar{P}_{d}\left(\gamma_{0}\right)$ will be obtained as a function of $\beta$, thus enabling the performance analysis of ED for any arbitrary primary power variability. The exact value of $\beta$ can be assumed to be unknown to the secondary receiver due to the inability of a secondary user to perfectly know beforehand the primary signals that might be present in the sensed band and their properties. This parameter will henceforth be referred to as signal uncertainty. The validity of this model is corroborated in Figure 3. For the cases where the received SNR can be approximated by Rayleigh distributions (Figure 3(a)) or gamma distributions (Figure 3(b)), the model of (6) provides a reasonable fit. Moreover, it is interesting to mention that, for some cases where the empirical value of $\beta$ is neither 0.27 (Rayleigh case) nor 0.5 (gamma case), the proposed model is able to provide more accurate fits, as illustrated in the example of Figure 3(c). In a few particular cases none of the considered approximations is perfect although all of them are able to qualitatively describe the empirical SNR distribution (Figure $3(d)$ ). In conclusion, the model of (6) can be employed to mathematically describe, in an approximated but acceptable manner, the empirical distribution of received SNR values.

\section{Approximation for the probability of detection}

To facilitate the analytical resolution of (5) with the proposed SNR PDF models, the term $P_{d}(\gamma)$ is approximated as follows. According to (4), $P_{d}(\gamma)$ can be expressed as:

$$
P_{d}(\gamma)=\mathcal{Q}(\zeta(\gamma))
$$

where $\zeta(\gamma)$ is given by:

$$
\begin{aligned}
\zeta(\gamma) & =\frac{\alpha \mathcal{Q}^{-1}\left(P_{f a}\right) \sqrt{2 N}-N(\gamma+1-\alpha)}{\sqrt{2 N}(1+\gamma)} \\
& \approx \alpha \mathcal{Q}^{-1}\left(P_{f a}\right)-\sqrt{\frac{N}{2}}(\gamma+1-\alpha)
\end{aligned}
$$

The Gaussian $Q$-function can be approximated by a secondorder exponential function:

$$
\mathcal{Q}(x) \approx e^{-\left(a x^{2}+b x+c\right)}, \quad x \geq 0
$$

with fitting coefficients $a=0.3845, b=0.7635$ and $c=$ 0.6966 [25]. Notice that $\zeta(\gamma)$, the argument of the $Q$-function in (7), may take both positive and negative values even though $\gamma \geq 0$ (see (8)). Since the approximation in (9) is valid for positive arguments only, the property $\mathcal{Q}(x)=1-\mathcal{Q}(-x)$ must therefore be used for negative values of $\zeta(\gamma)$. Applying this approximation to (7) it finally yields:

$P_{d}(\gamma)=\mathcal{Q}(\zeta(\gamma)) \approx\left\{\begin{array}{cc}e^{-\left(a[\zeta(\gamma)]^{2}+b \zeta(\gamma)+c\right)} & =e^{-\left(\Omega \gamma^{2}+\Psi \gamma+\Phi\right)}, \\ 1-e^{-\left(a[\zeta(\gamma)]^{2}-b \zeta(\gamma)+c\right)} & \zeta(\gamma) \geq 0 \\ =1-e^{-\left(\Omega \gamma^{2}+\Xi \gamma+\Theta\right)}, & \zeta(\gamma) \leq 0\end{array}\right.$

where $\zeta(\gamma) \geq 0 \Rightarrow \gamma \leq \xi, \zeta(\gamma) \leq 0 \Rightarrow \gamma \geq \xi$, and:

$$
\begin{aligned}
\Omega= & \frac{a N}{2} \\
\Psi= & -a \alpha \mathcal{Q}^{-1}\left(P_{f a}\right) \sqrt{2 N}-a N(\alpha-1)-b \sqrt{\frac{N}{2}} \\
\Xi= & -a \alpha \mathcal{Q}^{-1}\left(P_{f a}\right) \sqrt{2 N}-a N(\alpha-1)+b \sqrt{\frac{N}{2}} \\
\Phi= & a\left[\alpha \mathcal{Q}^{-1}\left(P_{f a}\right)+\sqrt{\frac{N}{2}}(\alpha-1)\right]^{2} \\
& +b\left[\alpha \mathcal{Q}^{-1}\left(P_{f a}\right)+\sqrt{\frac{N}{2}}(\alpha-1)\right]+c \\
\Theta= & a\left[\alpha \mathcal{Q}^{-1}\left(P_{f a}\right)+\sqrt{\frac{N}{2}}(\alpha-1)\right] \\
& -b\left[\alpha \mathcal{Q}^{-1}\left(P_{f a}\right)+\sqrt{\frac{N}{2}}(\alpha-1)\right]+c \\
\xi= & \alpha \sqrt{\frac{2}{N}} \mathcal{Q}^{-1}\left(P_{f a}\right)+\alpha-1
\end{aligned}
$$




$$
\begin{aligned}
\bar{P}_{d}^{M G}\left(\gamma_{0}\right)= & \int_{\gamma} P_{d}(\gamma) f_{\gamma}^{M G}(\gamma) d \gamma \\
\approx & \frac{\kappa}{\sqrt{2 \pi} \sigma_{\gamma}}\left[\int_{0}^{\xi} e^{-\left(\Omega \gamma^{2}+\Psi \gamma+\Phi\right)} e^{-\frac{1}{2}\left(\frac{\gamma-\gamma_{0}}{\sigma_{\gamma}}\right)^{2}} d \gamma+\int_{\xi}^{\infty}\left[1-e^{-\left(\Omega \gamma^{2}+\Xi \gamma+\Theta\right)}\right] e^{\left.-\frac{1}{2}\left(\frac{\gamma-\gamma_{0}}{\sigma_{\gamma}}\right)^{2} d \gamma\right]}\right. \\
\approx & \left.\frac{\kappa}{2} \operatorname{erfc}\left(\frac{\xi-\gamma_{0}}{\sqrt{2} \sigma_{\gamma}}\right)+\frac{\kappa}{2} \sqrt{\frac{1}{2 \sigma_{\gamma}^{2} \widetilde{\Omega}}} \exp \left(\frac{\widetilde{\Psi}^{2}}{4 \widetilde{\Omega}}-\widetilde{\Phi}\right)\left[\operatorname{erf}\left(\frac{2 \widetilde{\Omega} \xi+\widetilde{\Psi}}{2 \sqrt{\widetilde{\Omega}}}\right)-\operatorname{erf}\left(\frac{\widetilde{\Psi}}{2 \sqrt{\widetilde{\Omega}}}\right)\right]\right] \\
& -\frac{\kappa}{2} \sqrt{\frac{1}{2 \sigma_{\gamma}^{2} \widetilde{\Omega}}} \exp \left(\frac{\widetilde{\Xi}^{2}}{4 \widetilde{\Omega}}-\widetilde{\Theta}\right) \operatorname{erfc}\left(\frac{2 \widetilde{\Omega} \xi+\widetilde{\Xi}}{2 \sqrt{\widetilde{\Omega}}}\right) \\
\bar{P}_{d}^{R, G}\left(\gamma_{0}\right) \approx & \Upsilon\left(\gamma_{0}\right) \quad+\frac{1}{v^{2}} \frac{\bar{\Psi}}{4} \sqrt{\frac{\pi}{\bar{\Omega}^{3}}} \exp \left(\frac{\bar{\Psi}^{2}}{4 \bar{\Omega}}-\Phi\right)\left[\operatorname{erf}\left(\frac{\bar{\Psi}}{2 \sqrt{\bar{\Omega}}}\right)-\operatorname{erf}\left(\frac{2 \bar{\Omega} \xi+\bar{\Psi}}{2 \sqrt{\bar{\Omega}}}\right)\right] \\
+ & \frac{1}{v^{2}} \frac{\bar{\Xi}}{4} \sqrt{\frac{\pi}{\bar{\Omega}^{3}}} \exp \left(\frac{\bar{\Xi}^{2}}{4 \bar{\Omega}}-\Theta\right) \operatorname{erfc}\left(\frac{2 \bar{\Omega} \xi+\bar{\Xi}}{2 \sqrt{\bar{\Omega}}}\right)+\frac{1}{v^{2}} \frac{e^{-\Phi}-e^{-\left(\bar{\Omega} \xi^{2}+\bar{\Psi} \xi+\Phi\right)}-e^{-\left(\bar{\Omega} \xi^{2}+\bar{\Xi} \xi+\Theta\right)}}{2 \bar{\Omega}}
\end{aligned}
$$

\section{Closed-form expressions}

Substituting the approximations of (6) and (10) into (5) yields (12), where $\Omega=\Omega+1 / 2 \sigma_{\chi}^{2}, \widetilde{\Psi}=\Psi-\gamma_{0} / \sigma_{\gamma}^{2}$, $\widetilde{\Xi}=\Xi-\gamma_{0} / \sigma_{\gamma}^{2}, \widetilde{\Phi}=\Phi+\gamma_{0}^{2} / 2 \sigma_{\gamma}^{2}, \widetilde{\Theta}=\Theta+\gamma_{0}^{2} / 2 \sigma_{\gamma}^{2}$ and $\operatorname{erfc}(\cdot)$ is the complementary error function $[16,(7.1 .2)]$. The two last terms of (12c) lead to similar numerical values and approximately cancel out each other. The result of (12) could therefore be simplified to:

$$
\bar{P}_{d}^{M G}\left(\gamma_{0}\right) \approx \frac{\kappa}{2} \operatorname{erfc}\left(\frac{\xi-\gamma_{0}}{\sqrt{2} \sigma_{\gamma}}\right)=\frac{\operatorname{erfc}\left(\frac{\xi-\gamma_{0}}{\sqrt{2} \sigma_{\gamma}}\right)}{1+\operatorname{erf}\left(\frac{\gamma_{0}}{\sqrt{2} \sigma_{\gamma}}\right)}
$$

This approximation is valid over a wide range of SNR values and tighter for high $N$ and low $P_{f a}$ values.

For comparison purposes, (5) was also solved when the SNR follows Rayleigh and gamma distributions. In such cases, the resulting detection probability is given by (13), where $\Upsilon\left(\gamma_{0}\right)=$ $\exp \left(-(\pi / 4)\left(\xi / \gamma_{0}\right)^{2}\right), v=\sqrt{2 / \pi} \gamma_{0}, \bar{\Omega}=\Omega+\pi / 4 \gamma_{0}^{2}, \bar{\Psi}=\Psi$ and $\bar{\Xi}=\Xi$ for the Rayleigh distribution, while $\Upsilon\left(\gamma_{0}\right)=(1+$ $\left.2 \xi / \gamma_{0}\right) \exp \left(-2 \xi / \gamma_{0}\right), v=\gamma_{0} / 2, \bar{\Omega}=\Omega, \bar{\Psi}=\Psi+2 / \gamma_{0}$ and $\bar{\Xi}=\Xi+2 / \gamma_{0}$ for the gamma distribution. As in (12c), the last terms of (13) approximately cancel out each other, leading to the approximation

$$
\bar{P}_{d}^{R}\left(\gamma_{0}\right) \approx \exp \left(-\frac{\pi}{4}\left(\frac{\xi}{\gamma_{0}}\right)^{2}\right)
$$

for the Rayleigh case and

$$
\bar{P}_{d}^{G}\left(\gamma_{0}\right) \approx\left(1+\frac{2 \xi}{\gamma_{0}}\right) \exp \left(-\frac{2 \xi}{\gamma_{0}}\right)
$$

for the gamma case.

Figure 4 compares (4), (12) and (13). The resulting probability of detection for primary signals whose received SNR can be modeled as a gamma distribution is lower than that obtained for primary signals with Rayleigh SNR distributions. This can be explained by the fact that $\sigma_{\gamma}^{2} \approx 0.27 \gamma_{0}^{2}(\beta \approx 0.27)$ for the Rayleigh case while $\sigma_{\gamma}^{2}=0.5 \gamma_{0}^{2}(\beta=0.5)$ for the gamma case. In other words, for a given average SNR, the gamma distribution is characterized by a higher variance. This suggests that a higher signal variability can therefore be associated to a lower detection performance. In fact, the results obtained with the modified Gaussian model indicate, under constant operating conditions, that the detection performance degrades as the primary signal variability $\beta$ increases $^{2}$, which is in accordance with the experimental results observed in Section III. In the opposite direction, (12) converges numerically to (4) as the signal variability decreases. This can also be predicted analytically since:

$$
\begin{aligned}
\lim _{\beta \rightarrow 0} \bar{P}_{d}^{M G}\left(\gamma_{0}\right) & =\lim _{\sigma_{\gamma} \rightarrow 0} \int_{\gamma} P_{d}(\gamma) \frac{\kappa}{\sqrt{2 \pi} \sigma_{\gamma}} e^{-\frac{1}{2}\left(\frac{\gamma-\gamma_{0}}{\sigma_{\gamma}}\right)^{2}} d \gamma \\
& =\int_{\gamma} P_{d}(\gamma) \delta\left(\gamma-\gamma_{0}\right) d \gamma=P_{d}\left(\gamma_{0}\right)
\end{aligned}
$$

where $P_{d}(\gamma)$ is given by (4) and $\delta(\cdot)$ is the Dirac delta function, which can be expressed as the limit of a Gaussian PDF as its standard deviation tends to zero. For a constantpower transmitter, the received SNR is constant (neglecting the channel propagation effects), meaning that the instantaneous SNR $\gamma$ equals the average value $\gamma_{0}$ at any time, and in such a case (4) and (12) coincide. It is also worth noting in Figure 4 that the proposed model with $\beta=0.27$ (which corresponds to a Rayleigh distribution), results in a detection probability that is quite similar to that obtained for Rayleigh SNR distributions, indicating that the proposed model is able to describe, with reasonable accuracy, the experimental probability of detection observed for real-world signals.

\footnotetext{
${ }^{2}$ Under sufficiently low SNR conditions, a higher signal variability implies a higher probability that the received signal power is above the decision threshold, and hence a higher detection probability as observed in the leftmost part of the curves in Figure 4. However, spectrum sensing is designed to operate in the regions of high detection probability (i.e., the right-most part of the curves), where the detection performance degrades as the signal variability increases. Therefore, from a practical viewpoint, an increased signal variability results in a degraded detection performance.
} 


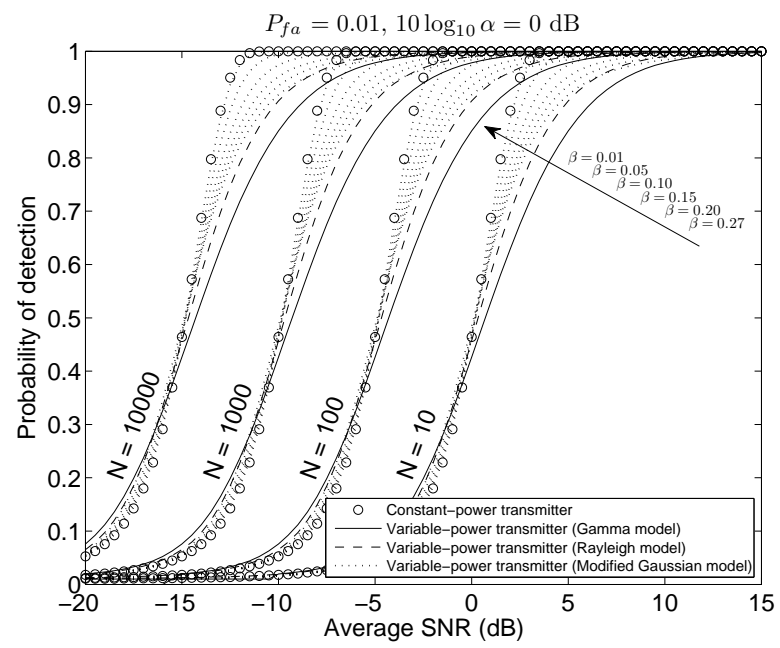

Fig. 4. Probability of detection for constant- and variable-power transmitters when the SNR is modeled as modified Gaussian $(\beta \in[0.01,0.27])$, Rayleigh and gamma distributions.

\section{E. Impact of the sensing period}

The model of (12) is able to describe the impact of the primary signal variability $\beta$ on the resulting detection performance of ED. However, the convergent trend observed in Figures 1 and 2 as the sensing period $N$ increases (discussed in Section III) is not described by (12). This phenomenon can be modeled as an effective reduction of the primary signal variability $\beta$ as $N$ becomes larger, which can be expressed as $\sigma_{\gamma}^{2}=\beta \tau(N) \gamma_{0}^{2}$, where $\tau(N) \in(0,1]$ is a monotonically decreasing function of $N$. An expression for $\tau(N)$ can be derived as follows. The ED test statistic $\mathbb{T}(\mathbf{y})$ as defined in (2) represents the received primary signal energy in a sensing interval of $N$ samples. The received signal power can therefore be obtained as $\mathbb{T}(\mathbf{y}) / N$. Notice that the term $|y[n]|^{2}$ in (2) is directly related to the instantaneous signal energy/power and the instantaneous SNR. Its variance $\operatorname{Var}\left(|y[n]|^{2}\right)=\operatorname{Var}\left(\left.[\mathbb{T}(\mathbf{y}) / N]\right|_{N=1}\right)$ is therefore associated to $\sigma_{\gamma}^{2}$, the variance of the instantaneous SNR. The reduction of the effective SNR variance as $N$ increases, $\tau(N)$, can therefore be determined based on the variance of the test statistic as:

$$
\tau(N)=\frac{\operatorname{Var}\left(\left.\frac{\mathbb{T}(\mathbf{y})}{N}\right|_{N \geq 1}\right)}{\operatorname{Var}\left(\left.\frac{\mathbb{T}(\mathbf{y})}{N}\right|_{N=1}\right)}
$$

Both variances are related as $[26,(2.21)]$ :

$$
\begin{aligned}
\operatorname{Var}\left(\frac{1}{N} \sum_{n=1}^{N}|y[n]|^{2}\right) & =\frac{1}{N^{2}} \sum_{n=1}^{N} \operatorname{Var}\left(|y[n]|^{2}\right) \\
& +\frac{1}{N^{2}} \sum_{\substack { m=1 \\
\begin{subarray}{c}{n=1 \\
n \neq m{ m = 1 \\
\begin{subarray} { c } { n = 1 \\
n \neq m } }\end{subarray}}^{N} \operatorname{Cov}\left(|y[m]|^{2},|y[n]|^{2}\right) \\
& =\operatorname{Var}\left(|y[n]|^{2}\right)\left(\frac{1}{N}+\frac{N-1}{N} \rho\right)
\end{aligned}
$$

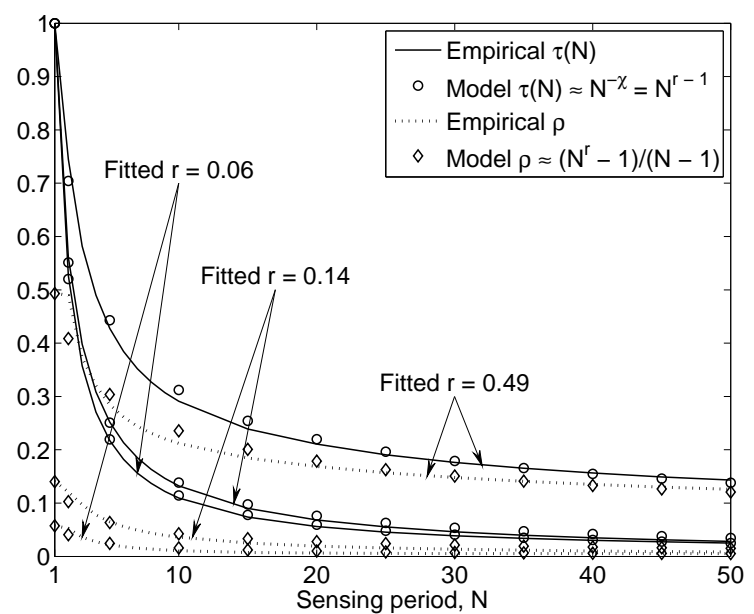

Fig. 5. Empirical and approximated results for $\rho$ and $\tau(N)$.

where it has been assumed that the samples $|y[n]|^{2}$ have equal variance (i.e., $\sigma_{\gamma}^{2}$ is constant along time) and $\rho$ represents the average correlation of distinct samples. According to (19), $\tau(N) \approx(1 / N)+\rho(N-1) / N$. In practice, however, the correlation coefficient $\rho$ is not completely independent of the sensing period $N$. Based on the empirical measurements performed in [9], and by means of curve fitting procedures, it was verified that the resulting empirical values of the average correlation coefficient can accurately be approximated as $\rho \approx\left(N^{r}-1\right) /(N-1)$, with $r$ being a fitting coefficient. Introducing this approximation into (19) yields $\tau(N) \approx N^{-\chi}$, with $\chi=1-r$, which was proven to describe experimental results reasonably well (see Figure 5). The parameter $\chi$ determines the rate at which the detection performance for various radio technologies converge as $N$ increases, and it will henceforth be referred to as the convergence rate. Notice that $\chi$ and $r$ are related to the correlation coefficient $\rho^{3}$, which means that the pace at which the detection performance for various radio technologies converge as $N$ increases in practice depends not only on the number of signal samples collected during the sensing period, $N$, but also on the correlation among them, which in turn depends on the sampling rate at which the samples are collected and its relation with the primary signal dynamics in the time domain. Let $T_{s}$ denote the sampling period (i.e., the time period between two consecutive signal samples). It is reasonable to assume that the correlation between two consecutive signal samples decreases as $T_{s} \rightarrow \infty$. If $T_{s}$ is sufficiently large to assume that $\rho \approx 0$, then $r \approx 0$ and $\tau(N) \approx 1 / N$, which is the maximum rate at which the detection performance can converge as $N$ increases $(\chi=1)$. On the other extreme, for completely correlated samples $(\rho \approx 1)$, which corresponds to $T_{s} \rightarrow 0, r \approx 1$ and $\tau(N) \approx 1$, meaning that in such a case the detection performance for various primary signals would not converge $(\chi=0)$, no matter how large the sensing period $N$ is (the trend would be as observed in Figure 4).

\footnotetext{
${ }^{3}$ In fact, it is interesting to note that $r=0$ for $\rho=0, r=1$ for $\rho=1$, and $r \rightarrow \rho$ as $N \rightarrow 1$.
} 


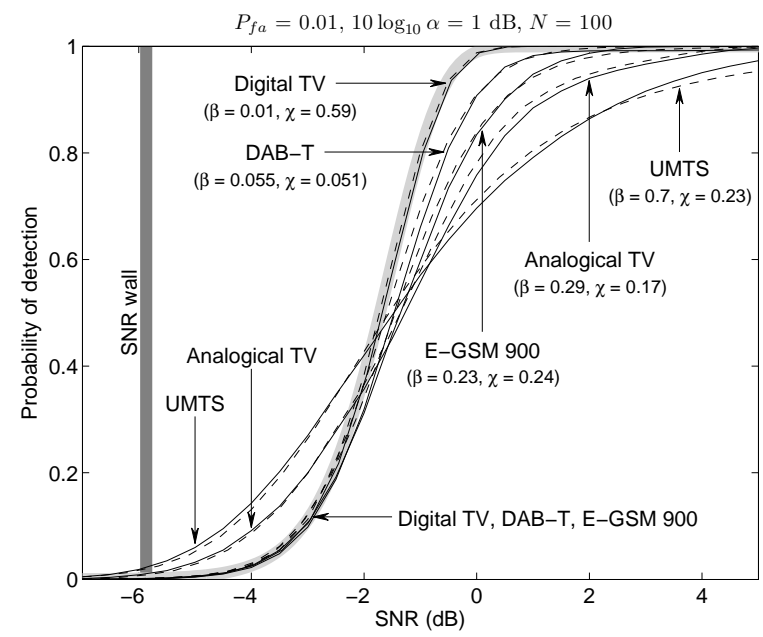

Fig. 6. Probability of detection as a function of the SNR: Classical theoretical performance (light thick line), experimental performance (solid line) and proposed model (dashed line).

Summarizing the results of this section, the detection performance of ED under variable primary transmission power patterns can be modeled as indicated by (12), where the average SNR $\gamma_{0}$ and the SNR variance $\sigma_{\gamma}^{2}$ are related as $\sigma_{\gamma}^{2}=\beta N^{-\chi} \gamma_{0}^{2}$. The signal uncertainty parameter, $\beta \geq 0$, represents the variability of the primary transmission power, while the convergence rate parameter, $\chi \in[0,1]$, determines the pace at which the detection performance for various primary signals converge as $N$ increases, and it is related to the correlation among the signal samples.

\section{F. Model validation}

The overall model is validated in Figure 6, where the experimental detection performance of ED is compared with the theoretical prediction of (4) as well as the performance predicted by the model of (12) based on the values of $\beta$ and $\chi$ derived from empirical measurements. The results are shown for $N=100$ and $1-\mathrm{dB}$ noise uncertainty. As opposed to the classical theoretical result of (4), which predicts the same detection performance for all the considered radio technologies, the proposed model is capable to capture and reproduce the impact of the variability of different transmission power patterns on the experimental performance of ED, thus providing a more accurate estimation of the real performance of ED in practice.

\section{Signal AND NOISE UNCERTAINTIES}

Based on the model developed in Section IV, this section analyzes the impact of the unknown primary power variability (signal uncertainty) on the detection performance of ED, with and without noise uncertainty, and compares the practical consequences of both degrading effects. The design and configuration of ED is discussed as well.

\section{A. Performance analysis without noise uncertainty}

The maximum interference constraint for a DSA/CR network can be mapped to a minimum detection probability requirement that must be satisfied for SNR values above a predefined thredhold, i.e. $\bar{P}_{d}\left(\gamma_{0}\right) \geq \bar{P}_{d}^{\text {min }}$ for all $\gamma_{0} \geq \gamma_{0}^{\text {min }}$. Let's assume that the DSA/CR system is designed to guarantee $\bar{P}_{d}^{\text {min }}=0.9$ at a given $\gamma_{0}^{\text {min }}$. This requirement implies a minimum sensing period for the DSA/CR network, $N^{\text {min }}$, which could be estimated as a function of $\gamma_{0}^{\text {min }}$ based on the classical result of (3) ( $\alpha=1$ in (4), assuming a perfectly cabrilated noise power), denoted as $N_{\text {classic }}^{\min }$. Based on this discourse, the equality $\bar{P}_{d}\left(\gamma_{0}\right)=\bar{P}_{d}^{\text {min }}$ should be true for $N=N_{\text {classic }}^{\min }$. To verify this statement, and based on the model of (12), Figure 7 plots the resulting $\bar{P}_{d}\left(\gamma_{0}\right)$ at the SNR values $\gamma_{0}=\gamma_{0}^{\min }$ for which $N_{\text {classic }}^{\text {min }}$ equals $10^{1}, 10^{2}, 10^{3}$ and $10^{4}$, when the target probability of detection is $\bar{P}_{d}^{\text {min }}=0.9$ and $N=N_{\text {classic }}^{\min }$. As it can be appreciated, when $N^{\text {min }}$ is computed based on (3), the equality $\bar{P}_{d}\left(\gamma_{0}\right)=\bar{P}_{d}^{\text {min }}$ holds for constant-power transmitters only $(\beta \rightarrow 0)$. For primary signals of uncertain power variability $(\beta>0)$, the resulting $\bar{P}_{d}\left(\gamma_{0}\right)$ is lower than the target $\bar{P}_{d}^{\text {min }}=0.9$. It is interesting to note that $\bar{P}_{d}\left(\gamma_{0}\right)$ first decreases below $\bar{P}_{d}^{\text {min }}$ as the primary signal variability $\beta$ increases, but after reaching a minimum value it increases again. If $\beta$ were made arbitrarily large, $\bar{P}_{d}\left(\gamma_{0}\right)$ would also increase. However, it is worth noting that the maximum signal variability observed empirically in the measurements performed in [9] was $\beta=2.29$. This indicates that for practical values of $\beta$, the resulting $\bar{P}_{d}\left(\gamma_{0}\right)$ is lower than $\bar{P}_{d}^{\text {min }}$ and the signal uncertainty phenomenon always is a degrading effect in practice, as illustrated in Figure 7 for various operating parameters. It is also interesting to note that the minimum value of $\bar{P}_{d}\left(\gamma_{0}\right)$ is reached at different values of $\beta$ (depending on the operating parameters), but it is always constant. In fact, it was verified that for $\bar{P}_{d}^{\text {min }} \geq 0.9$, the worst-case performance is $\min \left\{\bar{P}_{d}\left(\gamma_{0}\right)\right\} \approx 0.8 \cdot \bar{P}_{d}^{\text {min }}$, meaning that the worst detection probability is about $80 \%$ of the target value (this is however an optimistic estimation based on the no noise uncertainty assumption). This means that if signal uncertainty is not taken into account and the spectrum sensing function of a DSA/CR network is designed according to the classical result of (3), then there would be a potential and unacceptable risk of interference to the primary system.

\section{B. Performance analysis with noise uncertainty}

The results of Figure 7 correspond to the ideal case of no noise uncertainty, which is never true in reality. Let's consider that the DSA/CR devices are assumed to operate with a small noise power calibration error $\left(10 \log _{10} \alpha=0.1 \mathrm{~dB}\right)$ and $N^{\text {min }}$ is selected accordingly based on (4). In this case, the comparison of Figures 7 and 8 indicates that the detection performance degradation due to the signal uncertainty phenomenon may become more severe in the presence of noise uncertainty. The most unfavorable case corresponds to low convergence rates, where the worst-case detection performance decreases as $N$ increases (see Figure 8 ). This aspect should carefully be considered in the design of DSA/CR systems since DSA/CR devices are expected to operate in low SNR regimes, where the required sensing time intervals can usually be high, and in the unavoidable presence of noise uncertainty. 


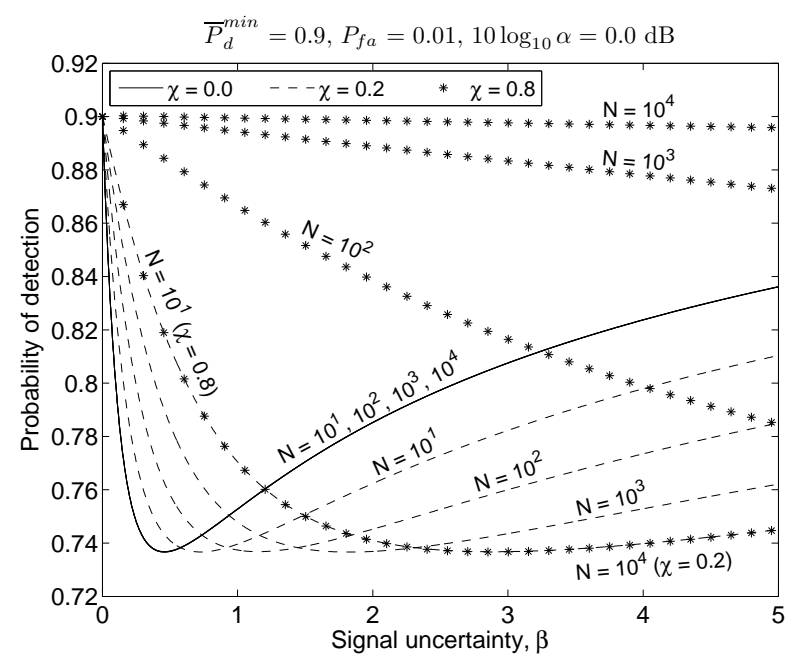

Fig. 7. Probability of detection as a function of the signal uncertainty for various convergence rates and sensing periods (without noise uncertainty).

The relative importance of noise and signal uncertainties on the detection performance of ED is illustrated in Figure 9. The operating point $(N=100$ and $\mathrm{SNR}=-2.92 \mathrm{~dB})$ has been selected according to (3) in order to provide a minimum detection performance of $\bar{P}_{d}^{\text {min }}=0.9$ for $P_{f a}=$ $0.01\left(10 \log _{10} \alpha=0.0, \beta=0\right)$. However, as appreciated, the real performance of $\mathrm{ED}$ is lower than the prediction provided by (3). In the presence of noise uncertainty only (10 $\left.\log _{10} \alpha=0.1, \beta=0\right)$, the detection probability decreases to $\bar{P}_{d}^{\text {min }}=0.86$. In the presence of signal uncertainty only $\left(10 \log _{10} \alpha=0.0, \beta=1\right)$, the detection performance experiences a more relevant degradation depending on the convergence rate parameter $\left(\bar{P}_{d}^{\text {min }}=0.80\right.$ for $\chi=0.5$ and $\bar{P}_{d}^{\text {min }}=0.74$ for $\left.\chi=0.2\right)$, and becomes more severe in the presence of noise uncertainty $\left(10 \log _{10} \alpha=0.1, \beta=1\right)$, down to $\bar{P}_{d}^{\text {min }}=0.76$ for $\chi=0.5$ and $\bar{P}_{d}^{\text {min }}=0.71$ for $\chi=0.2$.

The previous results indicate that the degrading effect of the signal uncertainty phenomenon can be of notable importance and becomes more severe in the presence of noise uncertainty, even for very small noise power calibration errors. This highlights the importance of carefully taking into account not only the noise uncertainty phenomenon but also the primary signal uncertainty in the design and configuration of spectrum sensing in real DSA/CR systems.

\section{Design and configuration of energy detection}

To effectively achieve the desired $\bar{P}_{d}^{\text {min }}$, the required sensing period $N^{\text {min }}$ should be computed taking into account not only the presence of noise uncertainty but also the potential primary signal variability (i.e., the signal uncertainty). To this end, the model of (12) constitutes a suitable tool and a more adequate alternative than the classical theoretical results of (3) and (4). Notice that (12) and its simplified version cannot be solved analytically for $N$ in closed form but they can be solved numerically in order to find the minimum sensing period required for a desired target performance under various operating conditions.

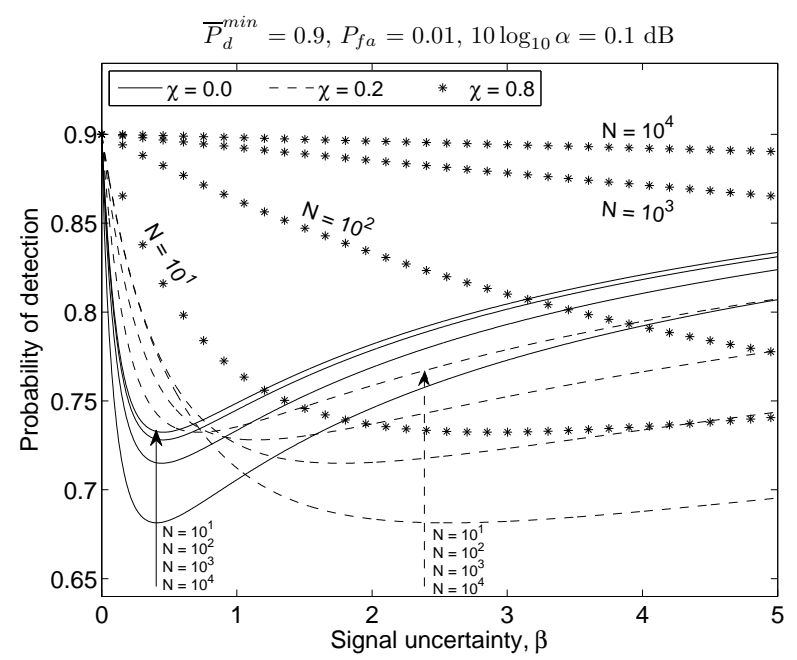

Fig. 8. Probability of detection as a function of the signal uncertainty for various convergence rates and sensing periods (with noise uncertainty).

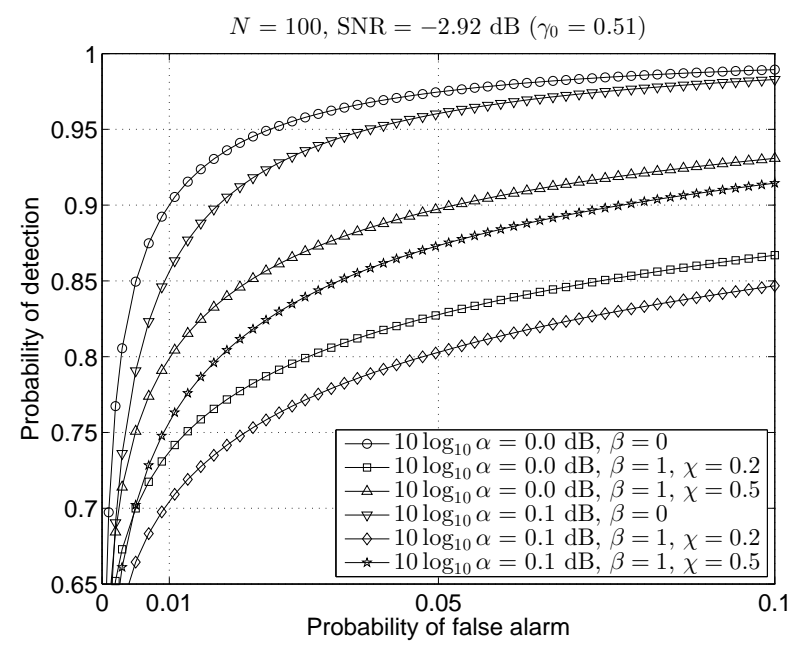

Fig. 9. Receiver operating characteristic of an energy detector under noise and signal uncertainties.

As an example, Figure 10 shows the $N^{\text {min }}$ required in a worst-case design (with and without noise uncertainty) as a function of the experienced $\gamma_{0}$ for a target $\bar{P}_{d}^{m i n}=0.9$ and assuming a maximum expected primary signal variability of $\beta=1.0$. The prediction of the classical theoretical result of (4) is also shown, indicating the SNR values $\gamma_{0}=\gamma_{0}^{\text {min }}$ for which $N_{\text {classic }}^{\min }$ equals $10^{1}, 10^{2}, 10^{3}$ and $10^{4}$. As it can be appreciated, the required $N^{\text {min }}$ should be increased with respect to the $N_{\text {classic }}^{\text {min }}$ prediction, for which it has been shown that $\bar{P}_{d}\left(\gamma_{0}\right) \leq \bar{P}_{d}^{\text {min }}$. However, the required increment depends on the convergence rate parameter $\chi$, being greater for lower convergence rates and vice versa. This can be explained by the fact that $\bar{P}_{d}\left(\gamma_{0}\right)$ increases with $N$, but faster for higher convergence rates $(\chi=0.8,1.0)$, in which case a lower increment of $N^{\text {min }}$ may be enough to meet $\bar{P}_{d}^{\text {min }}$. Since the convergence rate $\chi$ is related to the correlation among the primary signal samples ( $r$ or $\rho$ ), this means that the number of additional signal samples required for loosely correlated 

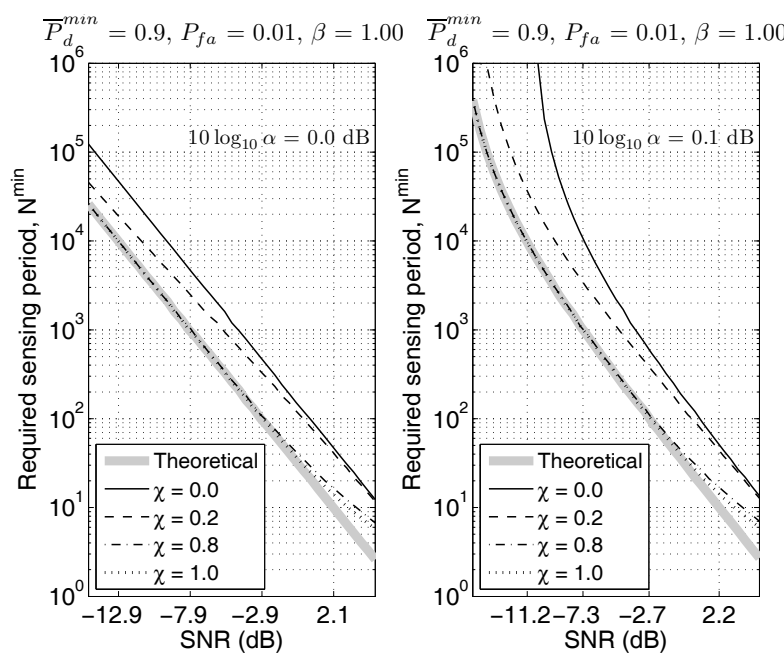

Fig. 10. Required $N^{\text {min }}$ as a function of the experienced SNR without $\left(10 \log _{10} \alpha=0.0 \mathrm{~dB}\right)$ and with $\left(10 \log _{10} \alpha=0.1 \mathrm{~dB}\right)$ noise uncertainty.

samples is lower than for highly correlated samples under the same operating conditions. In fact, Figure 7 indicates that a small number of loosely correlated samples $\left(N=10^{1}, \chi=\right.$ $0.8)$ may result in the same detection performance as a large number of highly correlated samples $\left(N=10^{4}, \chi=0.2\right)$. It is worth noting, however, that low values for the inter-sample correlation can be associated to large sampling periods $T_{s}$ as discussed in Section IV-D, meaning that the absolute sensing period in time units might require a significant increase (for both low and high correlation values) with respect to the value predicted by the classical theoretical results of (4). In the worst case, Figure 10 indicates an increase by a factor from 2 to 4 with respect to $N_{\text {classic }}^{\text {min }}$ under perfectly calibrated noise power and even several orders of magnitude for large sensing periods under noise uncertainty, where the impact of the signal uncertainty phenomenon becomes more severe. This numerical example not only highlights the importance of carefully taking into account both noise and signal uncertainties in the design of the spectrum sensing function of a DSA/CR network but also illustrates how the model proposed in this work can be used to adequately configure ED in real DSA/CR systems.

\section{CONCLUSION}

While the noise uncertainty phenomenon (inability to perfectly know the system noise properties to infinite precision) has widely been studied, the impact of the signal uncertainty concept introduced in this work (inability to perfectly know beforehand the primary signals that might be present in the sensed band and their properties) has not been taken into account in the analysis and performance evaluation of spectrum sensing in cognitive radio networks. In this context, and based on a generic mathematical expression for the received SNR distribution, this work has derived a closed form expression for the average detection probability under both noise and signal uncertainties, which has been employed to evaluate and analyze their impact on the detection performance of spectrum sensing. The obtained results have shown that the signal uncertainty phenomenon may result in significant performance degradations, particularly in the presence of noise uncertainty even for very small noise power calibration errors. Based on the proposed model, the design and configuration of spectrum sensing under both degrading effects has been discussed.

\section{APPENDIX \\ RELATION BETWEEN SIGNAL UNCERTAINTY AND PEAK-TO-AVERAGE POWER RATIO}

The energy detection performance model developed in this work quantifies the variability of a primary signal by means of the signal uncertainty parameter, defined as $\beta=\sigma_{\gamma}^{2} / \gamma_{0}^{2}$, where $\gamma_{0}$ and $\sigma_{\gamma}^{2}$ represent the average SNR and the SNR variance, respectively. Determining the value of the $\beta$ parameter for a particular radio technology is not straightforward in general, which hinders the application of the developed model. Alternatively, the variability of a signal can also be characterized by means of the Peak-to-Average Power Ratio (PAPR), which is a well-known parameter for many radio technologies. This appendix derives a closed-form relation between the signal uncertainty parameter $\beta$ and the PAPR in order to facilitate the practical application of the developed model.

The PAPR, $\Pi$, is equivalent to the ratio of the peak SNR, $\gamma_{\max }$, to the average SNR, $\gamma_{0}$ (i.e., $\Pi=\gamma_{\max } / \gamma_{0}$ ). According to the SNR distribution model of (6), $\gamma$ is not upper-bounded (i.e., $\gamma_{\max } \rightarrow \infty$ ). However, a confidence interval can be defined such that the SNR $\gamma$ is below a certain maximum value $\gamma_{\max }$ with a sufficiently representative probability $\epsilon$ (e.g., $\epsilon=0.95$ or $\epsilon=0.99$ ). According to this criterion, a representative value for $\gamma_{\max }$ can be obtained by computing:

$$
\begin{aligned}
\operatorname{Prob}\left(\gamma \leq \gamma_{\max }\right) & =\int_{0}^{\gamma_{\max }} f_{\gamma}^{M G}(\gamma) d \gamma \\
& =\frac{\operatorname{erf}\left(\frac{\gamma_{\max }-\gamma_{0}}{\sqrt{2} \sigma_{\gamma}}\right)+\operatorname{erf}\left(\frac{\gamma_{0}}{\sqrt{2} \sigma_{\gamma}}\right)}{1+\operatorname{erf}\left(\frac{\gamma_{0}}{\sqrt{2} \sigma_{\gamma}}\right)}=\epsilon
\end{aligned}
$$

and then solving the resulting equation for $\gamma_{\max }$, which yields:

$$
\gamma_{\max }=\gamma_{0}+\sqrt{2} \sigma_{\gamma} \operatorname{erf}^{-1}\left(\epsilon-(1-\epsilon) \operatorname{erf}\left(\frac{\gamma_{0}}{\sqrt{2} \sigma_{\gamma}}\right)\right)
$$

Making use of the equality $\sigma_{\gamma}^{2}=\beta \gamma_{0}^{2}$, the relation between $\beta$ and the PAPR follows from (21):

$$
\Pi=\frac{\gamma_{\max }}{\gamma_{0}}=1+\sqrt{2 \beta} \operatorname{erf}^{-1}\left(\epsilon-(1-\epsilon) \operatorname{erf}\left(\frac{1}{\sqrt{2 \beta}}\right)\right)_{(22)}
$$

The result in (22) can be used to numerically determine characteristic values of the signal uncertainty parameter $\beta$ for various radio technologies based on their typical PAPRs.

\section{REFERENCES}

[1] S. Haykin, "Cognitive radio: brain-empowered wireless communications," IEEE Journal on Selected Areas in Communications, vol. 23, no. 2, pp. 201-220, Feb. 2005.

[2] I. F. Akyildiz, W.-Y. Lee, M. C. Vuran, and S. Mohanty, "A survey on spectrum management in cognitive radio networks," IEEE Communications Magazine, vol. 46, no. 4, pp. 40-48, Apr. 2008.

[3] M. López-Benítez, "Cognitive radio," in Heterogeneous cellular networks: Theory, simulation and deployment. Cambridge University Press, 2013, ch. 13, (in press). 
[4] A. Ghasemi and E. S. Sousa, "Spectrum sensing in cognitive radio networks: requirements, challenges and design trade-offs," IEEE Communications Magazine, vol. 46, no. 4, pp. 32-39, Apr. 2008.

[5] T. Yücek and H. Arslan, "A survey of spectrum sensing algorithms for cognitive radio applications," IEEE Communications Surveys and Tutorials, vol. 11, no. 1, pp. 116-130, First Quarter 2009.

[6] D. D. Ariananda, M. K. Lakshmanan, and H. Nikookar, "A survey on spectrum sensing techniques for cognitive radio," in Proceedings of the Second International Workshop on Cognitive Radio and Advanced Spectrum Management (CogART 2009), May 2009, pp. 74-79.

[7] D. Noguet et al., "Sensing techniques for cognitive radio - state of the art and trends," Oct. 2009, IEEE SCC 41 P1900.6 White paper.

[8] H. Urkowitz, "Energy detection of unknown deterministic signals," Proceedings of the IEEE, vol. 55, no. 4, pp. 523-531, Apr. 1967.

[9] M. López-Benítez, F. Casadevall, and C. Martella, "Performance of spectrum sensing for cognitive radio based on field measurements of various radio technologies," in Proceedings of the 16th European Wireless Conference (EW 2010), Apr. 2010, pp. 1-9.

[10] R. Tandra and A. Sahai, "SNR walls for signal detection," IEEE Journal Selected Topics Signal Processing, vol. 2, no. 1, pp. 4-17, Feb. 2008.

[11] _ "SNR walls for feature detectors," in Proceedings of the 2nd IEEE International Symposium on New Frontiers in Dynamic Spectrum Access Networks (DySPAN 2007), Apr. 2007, pp. 559-570.

[12] F. F. Digham, M.-S. Alouini, and M. K. Simon, "On the energy detection of unknown signals over fading channels," IEEE Transactions on Communications, vol. 55, no. 1, pp. 21-24, Jan. 2007.

[13] A. Mariani, A. Giorgetti, and M. Chiani, "Effects of noise power estimation on energy detection for cognitive radio applications," IEEE Transactions on Communications, vol. 59, no. 12, pp. 3410-3420, Dec. 2011.

[14] J. J. Lehtomaki, M. Juntti, H. Saarnisaari, and S. Koivu, "Threshold setting strategies for a quantized total power radiometer," IEEE Signal Processing Letters, vol. 12, no. 11, pp. 796-799, Nov. 2005.

[15] M. López-Benítez and F. Casadevall, "Improved energy detection spectrum sensing for cognitive radio," IET Communications, Special issue on Cognitive Communications, vol. 6, no. 8, pp. 785-796, May 2012.

[16] M. Abramowitz and I. A. Stegun, Handbook of mathematical functions with formulas, graphs, and mathematical tables, 10th ed. New York: Dover, 1972.

[17] A. Sonnenschein and P. Fishman, "Radiometric detection of spreadspectrum signals in noise of uncertain power," IEEE Transactions on Aerospace and Electronic Systems, vol. 28, no. 3, pp. 654-660, Jul. 1992.

[18] A. Sahai, N. Hoven, and R. Tandra, "Some fundamental limits on cognitive radio," in Proceedings of the Forty-second Allerton Conference on Communications, Control, and Computing (Allerton Conference 2004), Sep. 2004, pp. 1-10.

[19] S. M. Kay, Fundamentals of statistical signal processing: detection theory. Prentice Hall, 1998, vol. 2

[20] H. V. Poor, An introduction to signal detection and estimation, 2nd ed. Springer, 1998, vol. 2 .

[21] D. Cabric, A. Tkachenko, and R. W. Brodersen, "Experimental study of spectrum sensing based on energy detection and network cooperation," in Proceedings of the First International Workshop on Technology and Policy for Accessing Spectrum (TAPAS 2006), Aug. 2006, pp. 1-8.

[22] — - "Spectrum sensing measurements of pilot, energy, and collaborative detection," in Proceedings of the IEEE Military Communications Conference (MILCOM 2006), Oct. 2006, pp. 1-7.

[23] A. Papoulis and S. U. Pillai, Probability, random variables, and stochastic processes, 4th ed. Boston: McGraw-Hill, 2002.

[24] J. Lundén, "Spectrum sensing for cognitive radio and radar systems," Ph.D. dissertation, Department of Signal Processing and Acoustics, Helsinki University of Technology, Nov. 2009.

[25] M. López-Benítez and F. Casadevall, "Versatile, accurate and analytically tractable approximation for the Gaussian Q-function," IEEE Transactions on Communications, vol. 59, no. 4, pp. 917-922, Apr. 2011.

[26] D. Blumenfeld, Operations research calculations handbook. CRC Press, 2001.

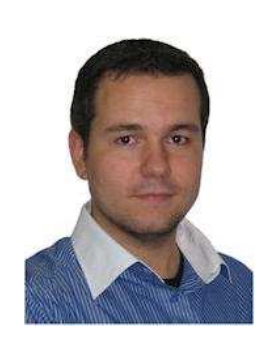

Miguel López-Benítez (S'08, M'12) received the B.Sc. (2003) and M.Sc. (2006) degrees in Communications Engineering (First-Class Honors) from Miguel Hernández University (UMH), Elche, Spain, and a Ph.D. degree (2011) in Communications Engineering from the Department of Signal Theory and Communications (TSC) of the Technical University of Catalonia (UPC), Barcelona, Spain.

He has been a Research Assistant in the Ubiquituous Wireless Communications Research (Uwicore) laboratory at UMH, and a Research and Teaching Assistant in the Mobile Communication Research Group (GRCM) at UPC. He currently is a Research Fellow in the Centre for Communication Systems Research (CCSR) at the University of Surrey, Guildford, Surrey, UK. His research interests include the field of mobile radio communication systems, with a special emphasis on radio resource management, heterogeneous wireless systems, quality of service provisioning, spectrum modeling and opportunistic/dynamic spectrum access in cognitive radio networks. He has been or is actively involved in the European-funded projects AROMA, NEWCOM++, FARAMIR, QoSMOS and CoRaSat along with Spanish projects COGNOS and ARCO. He has co-authored 2 book chapters and more than 40 papers in refereed journals and recognized conferences, and has been TPC for several IEEE conferences as well as reviewer for IEEE journals and conferences.

Dr. López-Benítez was the recipient of the 2003 and 2006 University Education National Awards, a distinction from the Spanish Ministry of Education and Science to the best national academic records, as well as some other distinctions from the Spanish professional association of telecommunications engineers. His M.Sc. thesis was awarded a national research prize from the France Telecom foundation (today Orange foundation) in the context of the 5th Archimedes University Competition, the main science contest for young researchers in Spain organized by the Spanish Ministry of Education and Science (+100 applications). He was shortlisted as a finalist for the IET Innovation Awards 2012 in the category of Telecommunications (+400 applications). For more details, please visit http://www.lopezbenitez.es.

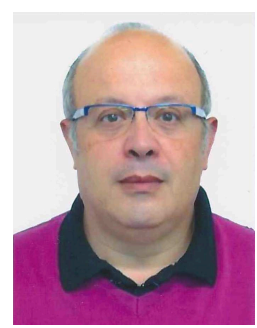

Fernando Casadevall (M'87) received the Engineer (1977) and Doctor Engineer (1983) degrees in telecommunications engineering from Universitat Politècnica de Catalunya (UPC), Barcelona, Spain.

In 1978, he joined UPC, where he was an Associate Professor from 1983 to 1991 . He is currently a Full Professor with the Department of Signal Theory and Communications, UPC. After graduation, he was concerned with equalization techniques for digital fiber-optic systems. He has also been working in the field of digital communications, with particular emphasis on digital radio and its performance under multipath propagation conditions. Over the last 15 years, he has been mainly concerned with the performance analysis and development of digital mobile radio systems. He has published around 150 technical papers in both international conferences and magazines, most of them corresponding to IEEE publications. His particular research interests include cellular and personal communication systems, multipath transceiver design (including software radio techniques), mobility, radio resource management, and end-to-end quality-of-service issues. During the last 15 years, he has participated in more than 30 research projects funded by both public and private organizations. In particular, he has actively participated in 15 research projects funded by the European Commission, being the Project Manager for three of them: Advanced Radio Resource management fOr Wireless Systems (ARROWS), Evolutionary Strategies for Radio Resource Management in Cellular Heterogeneous Networks (EVEREST), and Advanced Resource Management Solutions for Future All IP Heterogeneous Mobile Radio Environments (AROMA) (see http://www.gcr.tsc.upc.edu for details).

Prof. Casadevall has been a Technical Program Committee Member for different international IEEE supported conferences and a Reviewer for several IEEE magazines. From October 1992 to January 1996, he was in charge of the Information Technology Area, National Agency for Evaluation and Forecasting (Spanish National Research Council). 